ACTA UNIVERSITATIS LODZIENSIS

Folia Litteraria Romanica 16, 2021

https://doi.org/10.18778/1505-9065.16.12

\author{
Jean François Sablayrolles \\ Université Sorbonne Paris Cité \\ HTL UMR 7597 \\ jfsablayrolles@wanadoo.fr
}

\title{
Inventivité et renouvellement lexicaux (littéraire, argotique, etc.) pour une activité intemporelle
}

\begin{abstract}
RÉSUMÉ
Si les verbes et expressions dénommant l'acte sexuel en français académique sont rares (honorer une femme, plaisant, coüter, copuler didactiquess), il y en a pléthore en français non conventionnel. En sont exposés quelques-uns, transitifs directs (baiser, sauter...) et indirects (lui dégager les écoutilles...), mais aussi intransitifs (tirer son coup...), avec un point de vue masculin dominant. L'argot se renouvelle : bouillaver, niquer, pécho.... et se manifeste aussi une inventivité littéraire : faire catleya (Proust), jouer le jeu de la bête à trois dos (Jorif), acalifourcher (San-Antonio)... Deux chansons humoristiques peuvent clore ce florilège : Les ratés de la bagatelle (sous-titré la chose) et Les nuits d'une demoiselle, où fleurissent une multitude de verbes et expressions synonymes. Un néologisme sérieux est proposé par Bini Adamczak, féministe, circlusion, pour éviter la vision masculine incluse dans pénétration.
\end{abstract}

MOTS-CLÉS - français non conventionnel, inventivité littéraire, néologisme féministe, sexualité

\section{Inventiveness and Renewing the (Literary or Slang) Lexicon for an Atemporal Activity}

\section{SUMMARY}

In academic French, there are relatively few verbs or expressions referring to the sex act (honorer une femme is jocular, coiter, copuler didactic). There are, however, a large number in non-conventional French. Some of these are presented in this article, direct transitives (baiser, sauter...), indirect transitives (lui dégager les écoutilles...) and even some intransitives (tirer son coup...), all from a predominantly male point of view. Slang changes: bouillaver, niquer, pécho... and occurs also in literary inventiveness: faire catleya (Proust), jouer le jeu de la bête à trois dos (Jorif), acalifourcher (San-Antonio)... Two humourous songs close this selection: Les ratés de la bagatelle (subtitled la chose) and Les nuits d'une demoiselle, which teem with a multitude of synonymic verbs and expressions. One serious neologism has been proposed by the feminist philosopher Bini Adamczak, circlusion, to avoid the male viewpoint inherent in pénétration.

KEYWORDS - non-conventional French, literary inventiveness, feminist neology, sexuality 


\section{Introduction}

Un collègue reprochant à un étudiant d'avoir écrit dans une copie « il l'a baisée » cherche en vain un synonyme de baiser en français académique, et ne trouve que " il l'a honorée », emploi noté vieilli ou plaisant dans Le Petit Robert ( $P R$ désormais). Il aurait pu penser aussi, avec un même recul amusé, à connaître une femme, au sens biblique. Face à cette lacune ou indigence du français conventionnel ${ }^{1}$, pléthore de lexies (mots et expressions) disent l'acte sexuel en français non conventionnel (familier, vulgaire, argotique...) $)^{2}$.

Nous nous proposons d'étudier quelques verbes et expressions verbales dans lesquels se manifeste l'inventivité lexicale des locuteurs, en particulier dans l'argot et en littérature, entendue au sens large, mais pas seulement, par contraste avec des formulations plus anciennes dont nous présenterons d'abord certaines, parmi les plus usuelles, classées sur des critères syntaxiques.

\section{Dénominations de l'accomplissement de l'acte sexuel en français conventionnel et non conventionnel, ancien ou récent}

Dans la mesure où l'acte sexuel implique des partenaires, les verbes transitifs, directs ou indirects sont assez nombreux, comme l'indique Guiraud (1978a : 14) : "Pratiquement toutes les relations transitives peuvent ainsi servir de support à une image sexuelle. » Mais on trouve aussi des verbes intransitifs par lesquels nous commencerons.

\subsection{Des verbes intransitifs}

\subsection{1. « Didactiques »}

Au moins deux verbes intransitifs marqués « didactiques » sont attestés : coïter (didact. dans $P R$ ) et copuler (sans marque d'usage dans $P R$, mais la

\footnotetext{
${ }^{1}$ Cette pénurie n'est pas sans rappeler la chanson de Georges Brassens (1972) célébrant le sexe féminin et intitulé Le blason qui déplore « cette grande pitié de la langue française », mais l'auteur, malicieusement, dit « connaître les moyens de rendre hommage » au « plus bel apanage » du corps féminin qui n'a malheureusement pas de « joli nom chrétien », mais uniquement des termes vulgaires, « le pire de tous étant un vocable de trois lettres seulement qui dénomme aussi une foule de gens ».

2 Il est exclu de traiter dans cette communication les quelques « $1300 »$ synonymes recensés par Guiraud (1978a) pour le coït, qui deviennent 1500 dans Guiraud (1978b : 8) et « les près de 3000 mots et expressions pour désigner [la] même notion » qu'est l'acte sexuel (Guiraud 1978a : 13). L'auteur indique que ces relevés ne sont pas exhaustifs et aussi que « le vocabulaire de la sexualité se caractérise par une fécondité verbale tout à fait exceptionnelle.» (Guiraud 1978a : 13). Il montre ensuite que tous les verbes d'action, par exemple, sont susceptibles, selon le contexte, de prendre un sens sexuel.
} 
marque de domaine Zool est présente dans le Petit Larousse illustré, désormais $P L I)$. L'acte sexuel est " au sens propre [...] une 'relation', ainsi que le veut le verbe coïre 'aller avec' et son synonyme copulare 'joindre' », écrit Guiraud (1978a: 13).

\subsubsection{Par plaisanterie}

Le verbe forniquer est employé par plaisanterie ${ }^{3}$, à partir d'un emploi religieux pour le péché de chair, en particulier les relations sexuelles hors mariage ${ }^{4}$.

Le verbe pronominal s'accoupler employé pour les animaux est parfois étendu aux humains, en se teintant d'une valeur péjorative. L'utilisation de copuler pour des humains est marqué plaisant tant dans $P R$ que dans PLI. Les verbes spécifiques aux accouplements des animaux peuvent aussi, par extension, être utilisés pour les humains (beliner...), dans des emplois marqués et peu fréquents cependant.

\subsection{Des verbes transitifs directs}

Beaucoup plus nombreux sont les verbes transitifs directs, dont certains peuvent être éventuellement employés absolument.

\subsubsection{Français conventionnel}

En français conventionnel, les deux verbes prendre et posséder sont d'un emploi courant, d'un point de vue masculin, mais le premier semble être utilisé aussi par des femmes, plus que le second. Ce dernier a vu sons sens s'élargir pour signifier aussi 'être abusé, trompé', exactement comme le verbe baiser, en français non conventionnel.

\subsubsection{Français non conventionnel}

En français non conventionnel, le verbe le plus connu et sans doute le plus utilisé est en effet baiser, " euphémisme traditionnel qui semble dater du XVI ${ }^{e}$ siècle » (Guiraud 1978a : 154). Sa fréquence lui confère un aspect moins marqué que des emplois métaphoriques comme sauter, tringler, etc. On le retrouve sous des plumes masculines, comme celle de Georges Brassens (1966a), qui, dans

${ }^{3}$ Selon la marque d'usage présente dans $L e P R$.

${ }^{4}$ L'étymologie du mot est curieuse : il vient de fornix « voûte », parce que les prostituées romaines exerçaient leur activité dans des chambres voûtées. 
son Bulletin de santé, expliquait son amaigrissement par une autre raison qu'un cancer supposé par des médias qu'on n'appelait pas encore people:

Si j'ai quitté le clan des gros, des joufflus, des obèses,

C'est que je baise, que je baise, que je baise

Comme un bouc, un bélier, une bête, une brute

Je suis hanté le rut, le rut, le rut, le rut.

Ce verbe est aussi utilisé, entre autres, par une écrivaine, Virginie Despente, qui en a fait le titre de son premier livre paru en 1994 puis celui du film qu'elle en a tiré (2000), Baise-moi, mais son caractère inattendu et provocateur a joué un rôle d'accroche et fait beaucoup parler.

Le verbe niquer, originaire du sabir d'Afrique du Nord, de l'arabe nak, est daté de 1890 en français par $P R$, mais il s'est répandu plus tardivement et a connu une grande extension à partir de la fin $\mathrm{du} 20^{\mathrm{e}}$ siècle, en particulier comme terme d'insulte.

\subsubsection{Des verbes argotiques plus récents}

D'autres verbes argotiques plus récents sont recensés par Jean-Pierre Goudaillier (1997), alors qu'ils sont absents tant du dictionnaire de Guiraud que du PR 2020, ce qui est l'indice d'un renouvellement lexical dans le domaine. On relève ainsi bouillav(er), donné dans Wiktionnaire comme un terme argotique venant du romani et signifiant 'faire l'amour très vulgairement, très salement', fucker, emprunté à l'anglais, la construction transitive de partouser (qui n'est donné par $P R$ que comme intransitif), l'acception « coïter » de plomber, pounchave d'origine gitane, quène, verlan de niquer, réti, verlan de tirer (dont cette acception est présente dans $P R$, à partir de tirer son coup), tiser, zeub, verlan de baiser. La plupart de ces verbes sont transitifs directs mais susceptibles d'emplois absolus.

\subsection{Verbes transitifs indirects}

Le verbe sans doute le plus fréquent et assez neutre, quoique indiqué familier par $P R$ dans ce sens, est coucher avec. Comme pour d'autres verbes vus précédemment, il y a eu au cours du temps une spécification et restriction du sens, sans doute euphémique, puisque le verbe signifie étymologiquement 's'étendre', et on peut s'étendre à côté de quelqu'un pour simplement se reposer ou dormir. Mais le fait de partager régulièrement la couche de quelqu'un sous-entend que l'on a des relations sexuelles avec cette personne. C'est là l'origine de la dénomination concubin, lorsque l'union n'est pas sanctionnée par le mariage. 


\subsection{Expressions}

\subsubsection{Constructions intransitives}

L'expression s'envoyer en l'air est familière comme le sont aussi de nombreuses autres expressions parmi lesquelles tirer son coup, tirer sa crampe, tremper son biscuit, tremper son boudoir, tremper sa nouille, etc. Remarquons que si la première n'est pas genrée, de même que pratiquer / réaliser / utiliser / adopter la position du missionnaire (la synapsie est rarement utilisée avec un verbe), la plupart des autres expriment un point de vue masculin.

\subsubsection{Constructions transitives indirectes}

L'expression faire l'amour est courante en français contemporain mais le sens sexuel est relativement récent dans l'histoire de la langue. À l'époque classique, elle est encore souvent équivalent de " faire la cour », même si le sens moderne d' 'avoir des rapports sexuels' est daté de 1622, mais il ne se répand guère qu'au $19^{\mathrm{e}}$ siècle, d'après Rey (1992). Cette expression peut être employée avec un complément qui indique le partenaire et qui est introduit pas les prépositions à ou avec, avec une nuance de sens, mais elle est très souvent construite absolument.

L'expression faire un câlin (à), de type euphémique, est d'un emploi relativement récent, daté des années 1970/1980 dans Rey (1992).

Des métaphores sont reconnaissables dans lui dégager les écoutilles, employé dans le film Le bonheur est dans le pré d'Étienne Chatillez (1995) et dans lui ramoner la cheminée. Là encore le point de vue est masculin.

\section{Des manières ludiques et/ou inventives de « dire la chose » dans la littérature, au sens large, mais pas uniquement}

L'avant dernier exemple tiré d'un film fait passer de la langue et du lexique tel qu'il peut être décrit, hors contexte, si ce ne sont les exemples, dans les dictionnaires au discours, dans des productions effectives. Sauf pour le dernier point juste avant la conclusion, cette partie expose des innovations lexicales dénommant l'activité sexuelle prises dans la littérature au sens large, y compris la chanson.

\subsection{Une périphrase euphémique de Proust}

Pour éviter d'exprimer trop crûment le coït, deux personnages de $L a$ Recherche du temps perdu de Proust, Swann et Odette, recourent à la périphrase codée faire catleya (avec un seul -t-). Il ne semble pas qu'il y ait dans la création 
de cette expression, de métaphore. C'est un euphémisme codé qui caractérise ces personnages et les relations qu'ils entretiennent. Odette de Crécy aimait beaucoup ces fleurs.

\subsection{Richard Jorif}

Le roman de Richard Jorif Le Burelain (1989, éd. François Bourin) regorge de paléologismes, mots inconnus de la quasi-totalité de ses lecteurs pour être réintroduits dans la langue d'où ils avaient disparu. Ils produisent le même effet que des néologismes, mais aucun ne concerne directement la sexualité. Les vrais néologismes en revanche sont rares, mais il y en au moins deux en relation avec la sexualité et un mot rare où il le fait croire.

Le jeu de la bête à trois dos, dont le sens ne peut pas être compositionnel, est le détournement de l'expression connue, et utilisée, entre autres, par Georges Brassens (1966b) jouer le jeu de la bête à deux dos, avec le remplacement de deux par trois dans une situation de triolisme (mot pas très ancien puisque daté de 1985 dans $P R$ ) :

Frédéric balança ; mais curieux de savoir s'il était capable de jouer le jeu de la bête à trois dos, il revint les attendre la semaine suivante. Elles coururent à lui, excitées comme des paonnes. (p. 137)

S'éplucher la zuchette est la création d'une expression fondée sur une métaphore, la zuchette étant une variété de concombre, pour dénommer l'onanisme auquel s'adonnent des personnages fictifs :

C'était à croire que chaque fois qu'un garçon paraissait dans un roman, il n'avait de cesse qu'il ne se fût épluché la zuchette avec une fureur répétitive. (p. 159)

L'emploi du mot rare vespériser peut induire les lecteurs en erreur en songeant à une pratique sexuelle tant il est vrai que tout verbe d'action peut signifier l'acte sexuel, alors que ce verbe signifie tout simplement 'réprimander, tancer, morigéner'. C'est d'ailleurs ce que pense la jeune fille de la fiction en disant :

Ah, voilà ! Vespérisée ! ça c'est un mot. Je le garde pour moi, pour les fois où je parle toute seule, ça m'arrive... Mais à part ça, il n'est pas utilisable. Vous me voyez dire à quelqu'un : « L'autre jour, Tourny [son chef de service] m'a vespérisée. » Je me demande ce qu'il imaginerait. (p. 4)

\subsection{Les inventions de Frédéric Dard dans San-Antonio}

Le relevé effectué par Walid Dekdouk dans sa thèse consacrée au vocabulaire de San Antonio (à partir des incipit de six romans) ne contient aucune autre création verbale pour signifier le coït que acalifourcher. Il relève en revanche 
beaucoup de lexies familières, argotiques ou innovantes pour les parties du corps et différentes pratiques sexuelles. La prise en compte de la totalité de l'œuvre livrerait sans aucun doute un matériel lexical abondant dans ce domaine.

\title{
2.4. En chanson
}

\subsubsection{Faire la chose}

La chanson «Les ratés de la bagatelle », écrite par Marc Berthomieu et qui a pour sous-titre « la chose», a comme premier vers de chaque strophe l'anaphore "Y a ceux qui font la chose » ou une variante qui comporte toujours le mot " chose » à la fin. Ce mot chose est employé, par sous-entendu, faussement euphémique et un peu grivois, pour l'acte sexuel, de type hétérosexuel. Pierre Guiraud (1978a : 23) a montré comment toute expression d'une action pouvait dire l'acte sexuel, qui est l'acte par excellence : «Toute 'action' contient virtuellement et à l'état latent l'image de l'acte sexuel qui, le contexte aidant, va spontanément se développer ».

Ce sont la répétition de la même formulation associant deux mots au sens vague, le décalage entre ces mots vagues et un référent précis que tout le monde devine ${ }^{5}$ et les différentes situations ou motivations du coït évoquées qui confèrent une incontestable tonalité comique à cette chanson que plusieurs chanteurs et chanteuses ont mise à leur répertoire, dont Patachou (Philips, 1959), en ligne :

\author{
Y a ceux qui font la chose \\ En regardant l'plafond \\ Ou en comptant les mouches \\ Qui sont su'l'guéridon \\ Y a ceux qui font la chose \\ En pensant à l'argent \\ Et ceux qui n'pensent à rien \\ Parce que c'est fatiguant \\ Y a ceux qui font la chose \\ En s'demandant pourquoi \\ Et ceux qui font la chose \\ Comme s'ils étaient en bois
}

\footnotetext{
5 À la différence d'être porté sur la chose qui est une expression de la langue lexicographiée comme telle dans $P R$, faire la chose est absent du PR, comme du Dictionnaire Hachette 2009, du Petit Larousse 2008, du Nouveau Littré 2006 et du Lexis. Sa présence dans le TLFi n'est accompagnée d'aucun exemple. Chose peut, par euphémisme, dénommer l'acte sexuel. Mais on peut facilement imaginer des contextes où le syntagme faire la chose n'aurait aucun lien avec la sexualité.
} 
Ceux qui pendant la chose

Se mettent à bégayer

Et qui s'étonnent après

D'avoir des quintuplés

Y a ceux qui font la chose

En chantant une chanson

En battant la mesure

En tapant du talon

Y a ceux qui font la chose

Toujours un peu distants

Parce que c'est l'seul moyen

Pour avoir des enfants

Ceux qui pendant la chose

Poussent des cris violents

Pour que les voisins croient

Qu'ils passent un bon moment

Y a ceux qui font la chose

Sans passion ni désir

Parce que c'est le seul truc

Qui fasse vraiment maigrir

Y a ceux qui font la chose

Pour battre des records

Tous ceux dont les appas

$\mathrm{Ne}$ sont qu'un livre d'or

Y a ceux qui font la chose

Avec des accessoires

Qu'ont besoin d'une trompette

Ou bien d'une balançoire

Ceux qui lorsque la chose

Est déjà terminée

Se demandent si la chose

Va bientôt commencer

Y a ceux qui font la chose

Pas pour les sensations

Une seule fois dans l'année

Pour les allocations

Ils ont l'œil triste le cou tendu

Les ratés de la bagatelle

Ils font la chose en pardessus

Les ratés du fruit défendu 
Car tous ceux à qui

La chose ne fait rien

N'aiment pas ceux à qui

La chose fait du bien

Source : https://www.youtube.com/watch?v=udtXnhovSsw, consulté le 7 octobre 2020

\subsubsection{Les nuits d'une demoiselle}

La chanson «Les nuits d'une demoiselle » écrite par Guy Breton, mise en musique par Raymond Legrand et interprétée par Colette Renard (disque Vogue, 1963), contient, à l'inverse de la précédente, pléthore d'expressions pour les relations sexuelles, présentes ou absentes du dictionnaire de Guiraud (1978a), aux sens plus ou moins directement compréhensibles, compositionnels, et/ ou métaphoriques mais le cotexte dicte le sens, même si on ne comprend pas nécessairement comment est faite la lexie ou l'expression.

Tout l'intérêt de la chanson réside précisément dans cette accumulation de formulations rares pour la plupart, qui surprennent et amusent, et aussi dans le contraste avec la brièveté de la chute, avec le seul mot je baise synonyme ou quasi synonyme de toutes les expressions qui précèdent. L'intérêt de cette chanson est également le point de vue féminin et féministe qui s'y manifeste, et qui contraste avec les points de vue masculins prédominants, même si le texte a été écrit par un homme.

Que c'est bon d'être demoiselle

Car le soir dans mon petit lit

Quand l'étoile Vénus étincelle

Quand doucement tombe la nuit

Je me fais sucer la friandise

Je me fais caresser le gardon

Je me fais empeser la chemise

Je me fais picorer le bonbon

Je me fais frotter la péninsule

Je me fais béliner le joyau

Je me fais remplir le vestibule

Je me fais ramoner l'abricot

Je me fais farcir la mottelette

Je me fais couvrir le rigondin

Je me fais gonfler la mouflette

Je me fais donner le picotin

Je me fais laminer l'écrevisse

Je me fais fouailler le cœur fendu

Je me fais tailler la pelisse

Je me fais planter le mont velu 
Je me fais briquer le casse-noisettes

Je me fais mamourer le bibelot

Je me fais sabrer la sucette

Je me fais reluire le berlingot

Je me fais gauler la mignardise

Je me fais rafraîchir le tison

Je me fais grossir la cerise

Je me fais nourrir le hérisson

Je me fais chevaucher la chosette

Je me fais chatouiller le bijou

Je me fais bricoler la cliquette

Je me fais gâter le matou

Et vous me demanderez peut-être

Ce que je fais le jour durant

Oh! cela tient en peu de lettres

Le jour, je baise, tout simplement.

Source: https://greatsong.net/paroles-les-nuits-dune-demoiselle-colette-renard, consulté le 7 octobre 2020

Dans cette direction du point de vue féminin - et comme s'il était tenu compte des suggestions anciennes de Guiraud 1978 b à ce sujet, mais c'est très probablement indépendant - un pas supplémentaire (et décisif ?) a été franchi par la création d'un néologisme féministe conscient et volontaire.

\subsection{Un néologisme féministe}

Un renversement lexical féministe a en effet été récemment proposé par Bini Adamczak (2016) dans la création du néologisme circlusion en remplacement de pénétration (plutôt que comme antonyme) est-il indiqué sur des sites en ligne. C'est une manière de présenter l'acte sexuel d'une manière non exclusivement masculine. Il doit y avoir un autre point de vue (acceptable des deux côtés, masculin et féminin, et surtout féminin).

Guiraud (1978b : 187-194) abordait en effet, dans le chapitre intitulé « Le statut sémiologique de la 'féminité' ", le fait que les femmes doivent recourir à un vocabulaire masculin, voire machiste, et n'hésite pas à écrire, p. 187, que, de ce point de vue, « La femme est une aliénée du langage, [...] pensée et langage sont d'origine masculine [...] Les femmes parlent donc un langage qui n'est pas le leur ». Il enchaîne, p. 189-190, avec une suggestion :

On peut essayer d'imaginer comment seraient construits une image, un langage de la libido vécus et inventés par des femmes et à travers l'expérience de leur corps, pour autant que la relation sexuelle restât le symbole de la relation transitive, mais sentie et voulue par un sujet féminin et un objet masculin. 
Une chronique dans Le Monde de Maia Mazaurette, 25 août 2019, développe également le fait que les stéréotypes ont été le fait d'hommes : " Si les docteurs et penseurs du passé avaient eu un clitoris, ils n'auraient pas trouvé le fonctionnement féminin 'compliqué'. Ils l'auraient trouvé normal, parce que cela aurait été leur normalité ».

Le néologisme féministe circlusion, inventé précisément pour répondre à des constatations de ce type, ne semble pas s'être encore beaucoup répandu. Mais on ne peut pour autant parler d'échec. Il faut parfois du temps à des inventions verbales pour s'imposer. L'avenir décidera du sort de cette suggestion. L'inconvénient de ce néologisme tient au fait qu'il se prête mal à la création d'un verbe ( ?circlure) si l'adjectif (circlusif) pose moins de problème.

\section{Conclusion}

L'inventivité verbale liée à la sexualité est foisonnante, qu'il s'agisse d'euphémismes, vrais ou feints, ou au contraire de manières de dire crûment avec des images explicites ce dont il s'agit. Ce foisonnement est lié à la place centrale qu'occupe la sexualité dans la vie humaine.

À ce sujet, l'humour constaté dans certaines créations lexicales, comme dans les chansons citées, ne doit pas surprendre. La sexualité ne va pas sans peur ni tabou, comme la psychanalyse l'a montré. Le rire est une manière de parler de ce qui fait peur en désamorçant les risques.

On remarque des voix féminines peu nombreuses mais assez réconfortantes dans un monde quelque peu machiste et souvent vulgaire.

On peut se demander enfin, en particulier à propos de la création de circlusion, si on peut changer le vocabulaire et si changer les mots fait changer les choses. Ce n'est pas toujours le cas, mais parfois ça le peut.

\section{Bibliographie}

ADAMCZAK, Bini (2016), « Come on. Diskussion : Über ein neues Wort, das sich aufdrängt und unser Sprechen über Sex revolutionieren wird », Analyse und kritik, nº614 (http://www.akweb. de/ak_s/ak614/04.htm). Traduction en français (2018), « Come on. Discussion sur un nouveau mot qui émerge et qui va révolutionner notre manière de parler de sexe » GLAD. (https://www. revue-glad.org/1401, consulté le 7 octobre 2020)

DEKDOUK, Walid (2019), L'évolution du lexique dans les romans de San-Antonio durant les 50 ans de la publication de son œuvre, thèse de doctorat, Université Paris 13

GUIRAUD, Pierre (1978b), Sémiologie de la sexualité, Paris, Payot

MAZAURETTE, Maia (2019), Chronique « Le sexe selon Maia », Le Monde, 25 août 


\title{
Dictionnaires
}

Dictionnaire Hachette (millésime 2009), Hachette

GOUDAILLIER, Jean-Pierre (1997), Comment tu tchatches! Dictionnaire du français contemporain des cités, Paris, Maisonneuve et Larose

GUIRAUD, Pierre (1978a), Dictionnaire érotique, Paris, Payot

Le nouveau Littré (millésime 2006), Garnier

Le Petit Robert (millésime 2019), sous la direction de Josette Rey-Debove et Alain Rey, éd. Le Robert

Lexis ([1979] 1992), Dictionnaire de la langue française, Larousse

REY, Alain (1992), Dictionnaire historique de la langue française, éd. Le Robert

\begin{abstract}
Albums
BERTHOMIEU, Marc (1959), Les ratés de la bagatelle (La chose). Interprété par Patachou. Disques Philips

BRASSENS, Georges (1966a), Le bulletin de santé, album XI, Philips 836 293-2, « Supplique pour être enterré sur la plage de Sète »

BRASSENS, Georges (1966b), Le Moyenâgeux, album XI, Philips 836 293-2, « Supplique pour être enterré sur la plage de Sète »

BRASSENS, Georges (1972), Le blason, album « Fernande » Philips 63321116

BRETON, Guy (auteur), et LEGRAND, Raymond (compositeur) (1963), Les nuits d'une demoiselle. Interprété par Colette Renard. Disques Vogue
\end{abstract}

Jean-François Sablayrolles - agrégé de grammaire, professeur honoraire de l'Université Sorbonne Paris Cité et membre de l'UMR CNRS 7597 (Histoire des théories linguistiques), a consacré ses recherches à la néologie. Il a été rédacteur et co-rédacteur de cinq monographies et auteur des ouvrages : La Néologie en français contemporain (2000), Les Néologismes (2003), Les néologismes. Créer des mots français aujourd'hui (2017), Comprendre la néologie (2019). Il a créé en 2007 et dirigé la revue Neologica avec J. Humbley. 